\title{
A Coxian Model for Channel Holding Time Distribution for Teletraffic Mobility Modeling
}

\author{
B. H. Soong and J. A. Barria
}

\begin{abstract}
In this letter, we derive an algebraic set of equations that examines the relationships between the cell residence times and the handoff call's channel holding time. When the cell residence times have an Erlang or Hyper-Erlang distribution, the channel holding times can be represented by a Coxian model. An algorithm is presented to compute the parameters of the equivalent Coxian model. The analytical models proposed in this letter provide a flexible framework for further studies into the optimization and performance evaluation aspects of teletraffic mobile systems.
\end{abstract}

Index Terms-Cell residence time, channel holding time, coxian distribution, mobility modeling.

\section{INTRODUCTION}

$\mathbf{F}$ UTURE Personal Communication Services (PCS) presents many challenging problems where it is necessary to consider the mobility of users in addition to traffic scenarios for the purpose of teletraffic performance analysis. One key characteristic of these systems is the channel holding time that allows one to determine important network design parameters such as the new call blocking probability and the hand-off call blocking probability.

Recently, Kobayashi et al. [5] found that the dwell times were log normally distributed from measurements of the GPS signals located on-board the vehicles (taxis). The vehicles' velocity followed a truncated Gaussian distribution, and the handoff rate per call was inversely proportional to the length of the cells. With field data, Jedrzycki et al. [4] showed that a log normal distribution fitted the channel holding time for cellular systems better than the exponential distribution. The evidence motivates us to review the assumptions that are widely used in the literature.

The cell residence time or dwell time is defined as the duration of time the mobile user is communicating with the base-station (BS). Zonoozi et al. [6] used the Gamma distribution to model the cell residence time. Other researchers, Orlik et al. [3], suggested using the Sum of Hyperexponential distribution for the cell residence time. The characterization of the cell residence time as a nonexponential distribution proved to be timely. In contrast to wireline networks, the channel holding time de-

Manuscript receivedApril 4, 2000. The associate editor coordinating the review of this letter and approving it for publication was Dr. K. C. Chen.. This work was supported in part by the British Council and Association of Commonwealth Universities for the Fellowship at Imperial College of Science and Medicine, London, U.K.

B. H. Soong is with Communications Engineering Division, School of EEE, Nanyang Technological University, Singapore 639798 (e-mail: bh.soong@ieee.org).

J. A. Barria is with the Department of Electrical and Electronic Engineering, Imperial College of Science, Technology and Medicine, Exhibition Road,

London SW7 2BT, UK (e-mail: j.barria@ic.ac.uk).

Publisher Item Identifier S 1089-7798(00)11518-2. pends on the mobility of the users. When a call is allocated a channel, it will occupy the channel until it is released either due to call completion or when the mobile user moves to another cell. Recently, Fang et al. [2] proposed a new mobility model called the Hyper-Erlang distribution model for cell residence time to approximate the log normal distribution of the channel holding time. In this letter, we have derived an algebraic set of equations that examines the relationships between the cell residence times and the handoff call's channel holding time. It was found that when the cell residence times have an Erlang or Hyper-Erlang distribution, the channel holding times can be represented by a Coxian model. This work represents our ongoing research on a simplified analytic model for the application of performance evaluation and resource management of future mobile cellular systems.

\section{SySTEM MODEL}

Let $t_{k}$ be the cell residence time, $t_{c}$ the call holding time and $r_{k}$ is the residual life time of the call holding time when the call completes the $k$ th handoff successfully. The timing diagram model is given in Fang et al. [2]. The random variable denoting the handoff call's channel holding time is $t_{h h}=\min \left(t_{k}, r_{k}\right)$. Let $f^{*}(s)$ and $f_{h h}^{*}(s)$ be the Laplace transform of the density functions of the random variables $t_{k}$ and $t_{h h}$, respectively. Their main result is embodied in the following theorem which is reproduced here.

Theorem 1: Given a PCS network with exponential call holding time $\mu>0$ and Possion new call arrival rate $\lambda$. For this PCS network, the Laplace transform of the density function of the handoff call channel holding time distribution is given by

$$
f_{h h}^{*}(s)=\frac{\mu}{s+\mu}+\frac{s}{s+\mu} f^{*}(s+\mu) .
$$

The determination of the handoff call channel holding time is related with the cell residence time through eqn. (1). Similarly, the new call channel holding time distribution can be related to the cell residence time distribution with the Residual Life Theorem. Without loss of generality, we will concentrate on the handoff call channel holding time distribution since the methodology is similar. Suppose the cell residence time is Erlang-distributed ${ }^{1}$ with parameters $(m, \eta)$ where $\eta>0$ and $m$ is a positive integer. The Laplace transform of density function of the handoff call channel holding time is given by,

$$
f_{h h}^{*}(s)=\frac{\mu}{s+\mu}+\frac{s}{s+\mu}\left(\frac{m \eta}{s+\mu+m \eta}\right)^{m} .
$$

\footnotetext{
${ }^{1}$ Laplace transform of the Erlang density function is $f^{*}(s)=(\mathrm{mm} / \mathrm{s}+$ $m \eta)^{m}$.
} 


\section{MAIN RESULTS}

The rational polynomial in eqn. (2), expressed as $f_{h h}^{*}(s)=$ $N(s) / D(s)$ is the focus here. The following lemma provides insight to the link between the Coxian model for the handoff call channel holding time and the Erlang distribution for the cell residence time.

Lemma: For any positive integer $m=1,2, \cdots$, with $\eta>0$ and $\mu>0$, the Laplace transform of the handoff call channel holding time $f_{h h}^{*}(s)$ can be expressed as,

$$
f_{h h}^{*}(s)=\frac{N(s)}{(s+m \eta+\mu)^{m}}
$$

where $N(s)$ is a $(m-1)$ th order polynomial.

$$
N(s)=a_{m-1} s^{m-1}+a_{m-2} s^{m-2}+\cdots+a_{1} s+a_{0} .
$$

Proof: For this lemma, we expand eqn. (2),

$$
\begin{aligned}
f_{h h}^{*}(s) & =\frac{\mu(s+\mu+m \eta)^{m}+s(m \eta)^{m}}{(s+\mu)(s+\mu+m \eta)^{m}} \\
& =\frac{N^{\prime}(s)}{D(s)(s+\mu)}=\frac{N(s)}{D(s)} .
\end{aligned}
$$

In order to prove (3), firstly we need to show that there is a pole-zero cancellation of $(s+\mu)$. For ease of visualization, we concentrate on the numerator and substitute the following variables $s^{\prime}=s+\mu$, and $m \eta=\gamma$.

$$
\begin{aligned}
N^{\prime}(s) & =\mu(s+\mu+m \eta)^{m}+s(m \eta)^{m}=\mu\left(s^{\prime}+\gamma\right)^{m}+s(\gamma)^{m} \\
& =s \gamma^{m}+\mu \sum_{i=0}^{m}\left(\begin{array}{c}
m \\
i
\end{array}\right) s^{i} \gamma^{m-i} .
\end{aligned}
$$

Here, we introduce the notation for the binomial coefficients $B_{m i}=\left(\begin{array}{c}m \\ i\end{array}\right)$ Using the fact that $B_{m 0}=1$ and rearranging the terms, we have

$$
N^{\prime}(s)=s^{\prime} \gamma^{m}+\mu \sum_{i=1}^{m} B_{m i} s^{i} \gamma^{m-i}
$$

Therefore $N^{\prime}(s)$ contains a common factor $s^{\prime}=(s+\mu)$, which will cancel the pole. Next, we need to show that $N^{\prime}(s)$ is not divisible by $(s+m \eta+\mu)$. Similarly, we let $s^{\prime}=(s+\mu+m \eta)$.

$$
\begin{aligned}
N^{\prime}(s) & =\mu(s+\mu+m \eta)^{m}+s(m n)^{m} \\
& =\mu s^{m}+\left(s^{\prime}-\mu-m \eta\right)(m n)^{m} \\
& =\mu s^{m}+(m n)^{m} s^{\prime}-(\mu+m \eta)(m n)^{m} .
\end{aligned}
$$

Since $m \eta>0$ and $\mu>0$, we have that $N^{\prime}(s)$ does not contain the root $-(\mu+m \eta)$. Therefore, $N(s)$ will be the numerator polynomial of order $(m-1) . D(s)$ contains $m$ multiple roots at location $-(\mu+m \eta)$.
In the following, we seek to obtain an expression for the coefficients in eqn. (4). From the mathematical equation above,

$$
\begin{aligned}
N(s) & =\gamma^{m}+\mu \sum_{i=1}^{m} B_{m i}(s+\mu)^{i-1} \gamma^{m-i} \\
& =\gamma^{m}+\mu \sum_{i=1}^{m} B_{m, i} \gamma^{m-i} \sum_{k=0}^{i-1} B_{i-1, k} s^{k} \mu^{i-1-k} \\
& =\gamma^{m}+\sum_{i=1}^{m} \sum_{k=0}^{i-1} B_{m, i} B_{i-1, k} \gamma^{m-i} \mu^{i-k} s^{k} \\
& =\gamma^{m}+\sum_{i=1}^{m} \sum_{k=0}^{i-1} a_{i k}^{m} s^{k}
\end{aligned}
$$

with $a_{i k}^{m}=B_{m, i} B_{i-1, k} \gamma^{m-i} \mu^{i-k}$. Also note that,

$$
N(s)=\gamma^{m}+\sum_{k=0}^{m-1} s^{k}\left(\sum_{j=k+1}^{m} a_{j k}^{m}\right) .
$$

A minor computational effort is required to calculate the coefficients $a_{i k}^{m}$. However, it is obvious that these coefficients can be further simplified and their values will depend on $\mu$ and $\gamma=m \eta$. By relating eqn. (6) with the coefficients of the numerator polynomial $N(s)$ in (4) we have,

$$
\begin{aligned}
& a_{0}=(m \eta)^{m}+\sum_{j=1}^{m} a_{j 0}^{m} \\
& a_{k}=\sum_{j=k+1}^{m} a_{j k}^{m},
\end{aligned}
$$

for $k=1, \cdots, m-1$.

The results indicate $m$ multiple poles for the rational polynomial of the handoff call channel holding time distribution. This is extremely convenient, as we can use the partial fraction result [7],

$$
\begin{aligned}
f_{h h}(s)= & \frac{B_{1}}{(s+m \eta+\mu)^{m}}+\frac{B_{2}}{(s+m \eta+\mu)^{m-1}}+\cdots \\
& +\frac{B_{m}}{(s+m \eta+\mu)}
\end{aligned}
$$

where

$$
B_{j}=\left.\frac{1}{(j-1) !} \frac{\partial^{j-1}}{\partial s^{j-1}}[N(s)]\right|_{s=-(m \eta+\mu)}
$$

for $j=1, \cdots, m$.

Next, we relate $B_{1}, \cdots, B_{m}$ with the coefficients $a_{0}, \cdots, a_{m-1}$ of the numerator polynomial $N(s)$. By recursively differentiating (10), it is not too difficult to show that the relationship is governed by the equation as shown at the bottom of the page, or rewritten in the matrix-vector form with the substitution $s=-(\mu+m \eta)$

$$
\mathbf{B}=\mathbf{S a} .
$$

Notice that the matrix $\mathbf{S}$ is invertable with a determinant of 1 . The upper triangular form of the matrix allows us to relate the $\mathbf{a}$ and $\mathbf{B}$ vectors uniquely without the need for matrix inversion.

Theorem 2: When the cell residence time is Erlang distributed with parameter $(m, \eta)$, the handoff call channel 
holding time distribution can be represented as a Coxian server with the following Laplace transform:

$$
f_{h h}^{*}(s)=\sum_{i=1}^{m} \beta_{1} \beta_{2} \cdots \beta_{i} \alpha_{i+1}\left(\frac{\mu+m \eta}{s+\mu+m \eta}\right)^{i}
$$

where $\beta_{i}+\alpha_{i}=1$, for $i=1, \cdots, m$.

Proof: By relating the same powers of the rational polynomials (9) and the related coefficients (12), we have by defining $B_{j}=A_{m-j+1}$,

$$
A_{j}=\beta_{1} \beta_{2} \cdots \beta_{j} \alpha_{j+1}(\mu+m \eta)^{j}
$$

for $j=1, \cdots, m$.

Note that $\alpha_{m+1}=1$, and $\beta_{1}=1$ since $\alpha_{1}=0$. Therefore the unknowns, $\beta_{1}, \cdots, \beta_{m}$ can be solved recursively given the coefficients $A_{j}$. A Coxian server ${ }^{2}$ which consists of $m$ exponential stages with service rate of $\mu+m \eta$ can be used to represent eqn. (12). The parameters $\beta_{i}$ can be precomputed without any difficulty. Furthermore, the advantage with this form is that one can obtain directly the inverse Laplace transform of the handoff call holding time distribution given the coefficients in (9).

\section{DISCUSSION}

The proposed Coxian model allows us to calculate the coefficients through the computational equations without resorting to partial fraction expansion. The inversion of the resulting Laplace transform to the density function is straightforward. With this approach, we maintain that no compromise was made on making the modeling more difficult to fit a distribution from field data. The parameters the user requires to fit the distribution are still the size $m$ of the Erlang distribution, the parameter $\mu$ and $\eta$. With the specification of these parameters for the Erlang distribution of the cell residence times, the other coefficients can be calculated. Our results directly carry forward to the Hyper-Erlang cell residence distribution. In this case, the handoff call's channel holding time distribution is separable in the $s$-domain. Therefore, our Coxian model consists of parallel branches.

$f_{h h}^{*}(s)=\sum_{\ell=1}^{M} \zeta_{\ell}\left[\frac{\mu}{s+\mu}+\frac{s}{s+\mu}\left(\frac{m_{\ell} \eta_{\ell}}{s+\mu+m_{\ell} \eta_{\ell}}\right)^{m_{\ell}}\right]$

with the constraint, $\sum_{\ell=1}^{M} \zeta_{\ell}=1$.It is conceivable to reduce the complexity of this parallel model. In fact, Cox [1] has shown

\footnotetext{
${ }^{2}$ With this server, only one of the stages can be occupied at a given time. Before entering the $i$ th stage, an independent choice is made with probability $\beta_{i}$ the customer will proceed to the $i$ th exponential service stage and with probability $1-\beta_{i}$ the customer will depart immediately.
}

that with his general model that no further generality can be introduced with feedback and feedforward concept. It is envisaged that we can further decompose the Coxian model which is useful for teletraffic performance studies.

\section{CONCLUSIONS}

In this letter, we derive an algebraic set of equations that examines the relationships between the cell residence times and the handoff call's channel holding time. When the cell residence times has an Erlang or Hyper-Erlang distribution, the channel holding times can be represented by a Coxian model. An algorithm is presented to compute the parameters of the equivalent Coxian model. The set of equations allows the determination of the Coxian parameters to be computed easily without matrix inversion. We argue that the Coxian model provides a more flexible framework to study the nonexponential distribution of channel holding time. It is intuitively appealing since it is scalable and becomes a viable framework for further studies into the teletraffic aspects of mobile networks. Our future work would be to study the Coxian model for real-time control of the channel resources or to calculate the appropriate parameters for the dimensioning of mobile networks.

\section{ACKNOWLEDGMENT}

The authors gratefully acknowledge the anonymous reviewers for their comments.

\section{REFERENCES}

[1] D. R. Cox, "A use of complex probabilities in the theory of stochastic processes," in Proc. Cambridge Philosophical Society, vol. 51, 1955, pp. 312-319.

[2] Y. Fang and I. Chlamtac, "Teletraffic analysis and mobility modeling of PCS networks," IEEE Trans. Commun., vol. 47, July 1999.

[3] P. V. Orlik and S. S. Rappaport, "A model for teletraffic performance and channel holding time characterization in wireless cellular communication with general session and dwell time distribution," IEEE J. Select. Areas Commun., vol. 16, June 1998.

[4] C. Jedrzycki and V. C. M. Leung, "Probability distribution of channel holding time in cellular telephony," in IEEE VTC'97, Atlanta, GA, May 1997.

[5] T. Kobayashi, N. Shinagawa, and Y. Watanabe, "Vehicle mobility characterization based on measurements and its application to cellular communication systems," IEICE Tran. Commun., vol. E82-B, no. 12, December 1999.

[6] M. M. Zonoozi and P. Dassaayake, "User mobility modeling and characterization of mobility patterns," IEEE J. Select. Areas Commun., vol. 15, no. 7, pp. 1239-1252, October 1997.

[7] L. Kleinrock, Queueing Systems: Theory, vol. 1. New York: Wiley, 1976

$$
\left[\begin{array}{c}
B_{1} \\
B_{2} \\
B_{3} \\
\cdot \\
\cdot \\
\cdot \\
B_{m}
\end{array}\right]=\left[\begin{array}{ccccccc}
B_{m-1,0} s^{m-1} & B_{m-2,0} s^{m-1} & . \cdot & B_{30} s^{3} & B_{20} s^{2} & B_{10} s & 1 \\
B_{m-1,1} s^{m-2} & B_{m-2,1} s^{m-2} & . & B_{31} s^{2} & B_{21} s & 1 & \\
B_{m-1,2} s^{m-3} & B_{m-2,2} s^{m-3} & . \cdot & B_{32} s & 1 & & \\
\cdot & \cdot & . \cdot & \cdot & & & \\
\cdot & B_{m-2, m-3} s & \cdot & & & & \\
B_{m-1, m-2} s & \cdot & & & & 0 &
\end{array}\right]\left[\begin{array}{c}
a_{m-1} \\
a_{m-2} \\
a_{m-3} \\
\cdot \\
\cdot \\
\cdot \\
a_{0}
\end{array}\right]
$$

